\title{
Myxome odontogène récidivant : à propos d'un cas
}

\author{
Colnard $A^{1}$, Cousty $S^{1}$, L'Homme $A^{1}$, Courtois $B^{1}$, Duran $D^{1}$, Lauwers $F^{2}$ \\ ${ }^{1}$ UFR d'Odontologie, Université Paul Sabatier, Toulouse III, France \\ ${ }^{2}$ Service de Chirurgie maxillo-faciale, CHU, Toulouse, France \\ vepir@hotmail.com
}

Les myxomes odontogènes sont des lésions bénignes, le plus souvent asymptomatiques, mais qui peuvent être particulièrement invasives. Leur prévalence varie de $0,04 \%$ à 3,7\% (Halfpenny 2000), ils représenteraient $3 \%$ à $6 \%$ des tumeurs odontogènes. La localisation osseuse concerne essentiellement la mandibule. De rares cas de lésions condyliennes ont été décrits (Halfpenny 2000). Les myxomes siégeant au maxillaire semblent être plus agressifs que les mysomes mandibulaire (Kaffe 1997). Le traitement est chirurgical et les récidives fréquentes : $31,2 \%$ à la mandibule, 80,9\% au maxillaire (Gassab 2007).

Le cas présenté est celui d'une jeune fille de dix-sept ans qui a consulté pour une douleur dentaire chronique. Cette patiente avait bénéficié sept ans auparavant de l'exérèse chirurgicale d'un volumineux myxome maxillaire. L'examen endobuccal met en évidence une tuméfaction vestibulaire de consistance osseuse en regard de la 14. Les tests de vitalité sur 14 sont négatifs. Le cliché rétro-alvéolaire montre une image lacunaire dans la région de l'apex de la 14 . La tomodensitométrie met en évidence une lésion radioclaire, polylobulée, aux limites nettes, avec des cloisons à angles vifs, soufflant la table osseuse vestibulaire et refoulant la limite inférieure du sinus maxillaire, sans envahissement des fosses nasales.

Le traitement chirurgical consiste en une énucléation tumorale. L'examen histopathologique de la pièce opératoire confirme le diagnostic de myxome. La patiente est régulièrement suivie pendant 3 ans (examen clinique, tomodensitométrique, imagerie par résonance magnétique). Une reconstruction du maxillaire droit par greffon pariétal est ensuite envisagée.

Le traitement des myxomes varie selon les auteurs. Certains préconisent une exérèse large des lésions, pour limiter le risque de récidive (Killey 1964), (Schmidseder 1978), (Deron 1996), d'autres une chirurgie conservatrice avec énucléation de la lésion (Harder 1978). Slootweg et Wittkampf proposent une attitude thérapeutique adaptée en fonction du site (exérèse large au maxillaire et énucléation à la mandibule) (Slootweg 1986). La phase chirurgicale est ensuite suivie d'une surveillance clinique et radiologique, dont la durée est variable (plusieurs mois à années), avant d'envisager la restauration par greffe osseuse autologue et éventuellement une solution implantaire (Yin 2007).

La difficulté de prise en charge des myxomes réside essentiellement dans le caractère souvent agressif et récidivant des lésions. 